\title{
A comparison of haemodynamic metrics and intraluminal thrombus burden in common iliac artery aneurysms
}

\author{
Lachlan J. Kelsey ${ }^{1,2}$, Janet T. Powell ${ }^{3}$, Paul E. Norman ${ }^{1,4}$, Karol Miller ${ }^{2,5}$ and Barry J. \\ Doyle $^{1,6,7}$ \\ ${ }^{1}$ Vascular Engineering Laboratory, Harry Perkins Institute of Medical Research, Perth, Australia. \\ ${ }^{2}$ Intelligent Systems for Medicine Laboratory, School of Mechanical and Chemical Engineering, The \\ University of Western Australia, Australia. \\ ${ }^{3}$ Vascular Surgery Research Group, Imperial College London, UK. \\ ${ }^{4}$ School of Surgery, The University of Western Australia, Australia. \\ ${ }^{5}$ Institute of Mechanics and Advanced Materials, Cardiff University, UK. \\ ${ }^{6}$ British Heart Foundation Centre for Cardiovascular Science, The University of Edinburgh, UK. \\ ${ }^{7}$ School of Mechanical and Chemical Engineering, The University of Western Australia, Australia.
}

\begin{abstract}
Aneurysms of the common iliac artery (CIAA) are most commonly seen in association with an abdominal aortic aneurysm (AAA). Isolated CIAAs, in the absence of an AAA, are uncommon. Similar to AAAs, CIAA may develop intraluminal thrombus (ILT). As isolated CIAAs have a contralateral common iliac artery for comparison, they provide an opportunity to study the haemodynamic mechanisms behind ILT formation.

In this study, we compared a large isolated CIAA and the contralateral iliac artery using computational fluid dynamics (CFD) to determine if haemodynamic metrics correlate with the location of ILT. We performed a comprehensive CFD study and investigated the residence time of platelets and monocytes, velocity fields, time-averaged wall shear stress (TAWSS), oscillatory shear index (OSI) and endothelial cell activation potential (ECAP). We then correlated these data to ILT burden determined with computed tomography (CT).

We found that high cell residence times, low TAWSS, high OSI and high ECAP all correlate with regions of ILT development. Our results show agreement with previous hypotheses of thrombus formation in AAA and provide insights into the computational haemodynamics of iliac artery aneurysms.
\end{abstract}

Key words: iliac aneurysm, computational fluid dynamics, wall shear stress, intraluminal thrombus

\section{INTRODUCTION}

An aneurysm is a localised dilation of an artery which is life threatening when ruptured. Aneurysms of the common iliac artery (CIAA) are most commonly seen in association with an abdominal aortic aneurysm (AAA). Large-scale population screening studies show that, for men aged 65 years, the prevalence of an AAA is approximately $2 \%$ [1]. In $25 \%$ of these cases, there are co-existent aneurysms in one or both common iliac arteries and in 7\% of these cases aneurysms also exist in the internal iliac arteries [2]. The true prevalence of isolated CIAAs is unclear. The underlying causes of most aneurysms in these medium-to-large arteries are unknown and specific pathological or genetic causes are generally only identified in a small number of cases [3]. With respect to CIAAs there is no strong evidence base for their management and the threshold diameter for intervention is often when the diameter exceeds $3.5-4 \mathrm{~cm}$ [4], or based solely on clinical opinion. Healthy common iliac arteries typically have diameters of approximately 1 to $1.5 \mathrm{~cm} \mathrm{[2].} \mathrm{Figure} 1$ shows the healthy anatomy of the iliac artery region compared to an aneurysmal case.

In the past decades, computational fluid dynamics (CFD) has emerged as a powerful and popular tool for the study of blood flow dynamics of aneurysms and other cardiovascular disease [5-8]. With appropriate boundary conditions and model assumptions, CFD can simulate the blood flow through 
any vessel of the body using patient-specific geometries, typically derived from computed tomography (CT). To date, much attention has been focused on the study of haemodynamics in AAA, yet very little to the computational modelling and assessment of CIAA. When compared with the recent progress of computationally-aided assessment of AAA rupture risk [9-11] and other cardiovascular disease [12-15], there is a need to improve our ability to assess the risk of CIAA. CIAA occur in some computational models of AAA (i.e. [16]), although they were not the focus of that research. The authors are only aware of one other study primarily concerned with the haemodynamics of CIAA [17], and this work intends to provide more momentum to this topic.

ILT is found in over 70\% of clinically-relevant AAA [5, 18]. In some structural studies, ILT is thought to be beneficial as it may dampen the pressure-wave impact on the wall of AAA and, therefore, potentially reducing the chance of rupture. However, in stark contrast, other studies show that ILT increases rupture risk as it is a potential source of proteolytic activity and an accelerated inflammatory response, tied to local wall thinning, weakening and hypoxia [19-21]. The direct relationship between ILT and AAA volume is supporting evidence for this latter theory [20, 22, 23]. Furthermore, the onset and growth of ILT is a continuous process that involves a large number of haemodynamic and biochemical stimuli including the clotting of platelets and other particles [20, 2224].

ILT morphology ranges from a structured, layered material, to a homogenous fluid like structure [25]; at a specific state of maturity ILT becomes a poroelastic material, whereby its porous structure influences fluid transport from the lumen to the underlying tissue [7]. However, this behaviour is not accounted for in rigid wall CFD studies. This is primarily due to variability of ILT mechanical properties [7, 25] and the necessity of MRI imaging to properly view/understand ILT structure [26]. Despite the uncertainty this brings to existing fluid models, the relationship between haemodynamic indices, artery inflammation and thrombotic susceptibility is continually explored.

Initially, the haemodynamic study of idealised AAA geometries illustrated how the detachment of flow occurs and develops in enlarging aneurysms [27-29], where this behaviour is characterised by localised regions of low and oscillatory wall shear stress (WSS). These observations are physically similar to the separation of the boundary-layer downstream of stenoses, where Raz et al. [30] showed the direct relationship between platelet recirculation time and both platelet activation and acetylated thrombin generation. Furthermore, Rayz et al. [31] observed thrombus deposition in certain regions of patient-specific intracranial geometries with an increase in residence time and low WSS. More recently, Boyd et al. found that AAA rupture tended to occur in thrombus afflicted regions with predicted flow recirculation and low WSS [32]. In addition, it has been shown that regions of low, oscillatory WSS provide a well-described mechanical stimulus that promotes the inflammatory process by inducing an oxidative response in endothelial vascular cells [33]. As a means to quantify this behaviour in transient simulations of blood flow, the time-averaged wall shear stress (TAWSS) and the oscillatory shear index (OSI) [34] are often used to measure the interaction between the flow field and the artery wall (i.e. [5, 35-38]). Doyle et al. showed, via a serial CFD study of a patientspecific AAA over 2.5 years, the direct spatial relationship between low TAWSS, local aneurysm expansion and localised in vivo ILT development [5]. However, it should also be noted that despite thrombotic susceptibility typically being associated with low TAWSS and high OSI, O'Rourke et al. [37] and Arzani et al. [38] identified that regions of low OSI had a good correlation with the site specificity of ILT within aneurysms.

Furthermore, the morphology of AAA and the haemodynamic behaviour it induces has also been shown to exhibit an activated state of coagulation and fibrinolysis [39]. Biasetti et al. [40] postulated that platelets are activated as they enter into an aneurysm, where they are enveloped by vortices produced at the neck and later become entrapped in recirculation zones. Platelets then preferentially attach to pre-existing ILT or to the distal regions of the vessel (AAA). This is supported by multiple anatomic studies of ILT, as it commonly occurs in the distal anterior region of AAA [23, 41-43]. In 2012, an integrated fluid-chemical approach for modelling ILT formation in AAA was introduced by Biasetti et al. which showed agreement with the site specificity of ILT formation and the movement of vortex structures [44]. 
In addition, Basciano et al. [45] assessed the residence times and surface-shear loads of platelets and white bloods cells in a patient-specific AAA sac using Eulerian-Lagrangian CFD simulation methodology to investigate the onset of ILT, supporting Biasetti's work and the generalised view that aneurysms entrap blood particles. The one-way Lagrangian methodology used by Basciano [45] implements a particle drag force, pressure gradient force and gravity force, to calculate the particle trajectories in the arterial flow field, as these forces are thought to provide a reasonable prediction of cell transport.

The gravity force may be ignored for particles where the fluid to particle density ratio is close to unity [46]. Furthermore, when the particle distance from the wall approaches the same order of magnitude as the particle diameter, the typical lift and drag equations break down and require modification to accurately resolve the particle behaviour in the 'near-wall' region [46, 47]. Hardman et al. [47] implemented near-wall-modified lift and drag forces for the discrete Lagrangian phase in a large eddy simulation (LES) in both ideal and patient-specific geometries, and found that particle residence time (PRT) in aneurysm sacs was greatly increased for sac sizes where the maximum diameter exceeded 1.8 times the inlet diameter; thus proposing a critical size beyond which monocyte infiltration and wall degradation is greatly increased.

Following these previous studies into ILT and AAA, the objective of this study was to determine if/how well haemodynamic stimuli computationally match the areas of ILT development in both the advanced and the early stages of aneurysmal disease in the common iliac arteries. In this study, we investigated the blood flow of a 91-year-old male patient who did not have AAA, but had a large left CIAA with a maximum anterior-posterior diameter of $8.4 \mathrm{~cm}$. The right common iliac artery was 3.4 $\mathrm{cm}$ in diameter. ILT was present in both the large left CIAA and the less aneurysmal right common iliac artery.

\section{METHODOLOGY}

\subsection{Three dimensional (3D) reconstruction and trimming}

Contrast-enhanced CT data (pixel size $=0.82 \mathrm{~mm}$; slice thickness $=1 \mathrm{~mm}$ ) of a 91-year-old male with a large CIAA was imported into Mimics v17 (Materialise, Belgium). We reconstructed the lumen into $3 \mathrm{D}$ and conservatively smoothed the resulting surfaces (see Figure 1) following our previous methods [5]. We extended the inlet surface by $120 \mathrm{~mm}$ (determined using Wood's unsteady entrance length method [48]) and each outlet by 10 times the outlet diameter to ensure flow was fully developed entering the supra-celiac (SC) aorta and that the outlet boundary conditions did not affect the haemodynamics in the vessels. The resulting reconstruction contained a number of the minor arteries branching from the internal iliac arteries; these minor arteries are often not-captured by CT and subsequently neglected in CFD studies of the normal or diseased aortic bifurcation. The patient also had a 'pocket' (pouch-like cavity), recessing off the posterior of the left CIAA lumen, which was expected to produce complex flow.
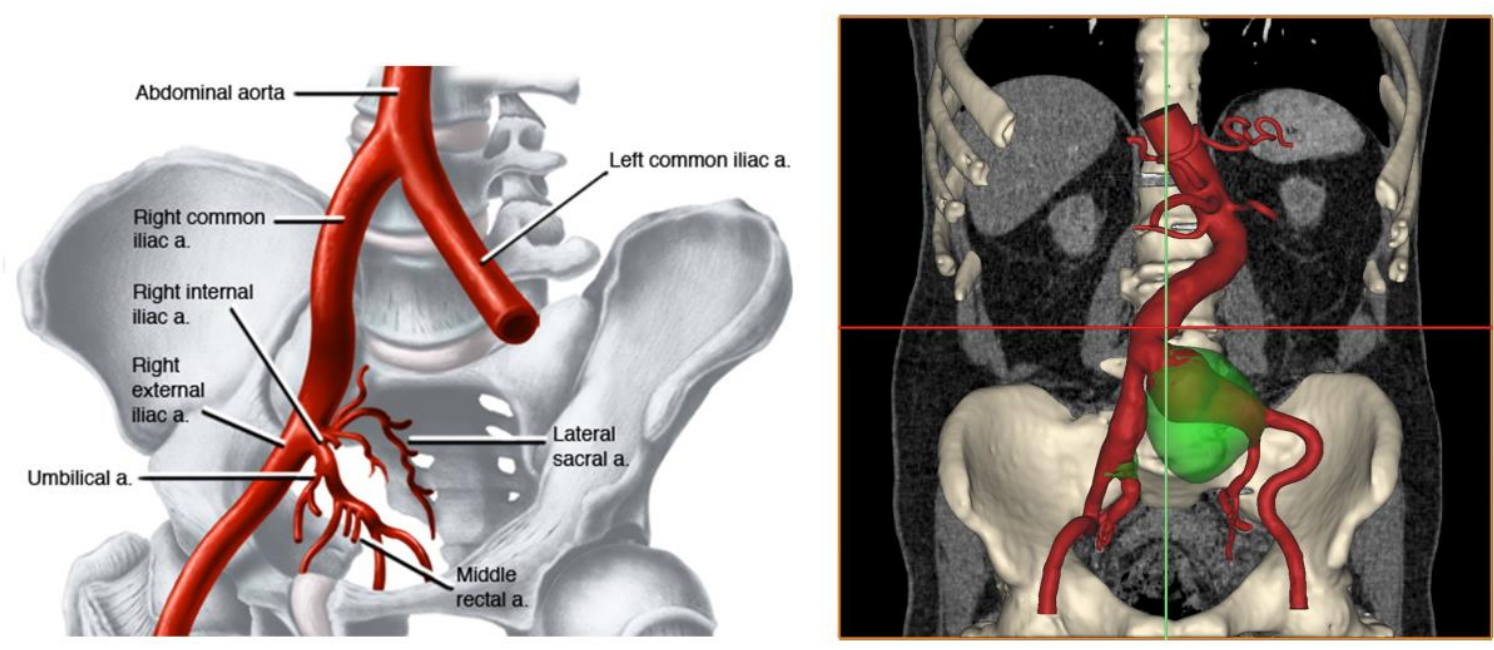
Figure 1: A diagram of normal pelvic vasculature [49] (Right) aside the coronal view of the patient's 3D vasculature - reconstructed from CT (Left). The patient's artery lumen is in red and thrombus in green. The patient's left CIAA had a maximum diameter of $8.4 \mathrm{~cm}(5.7 \mathrm{~cm}$ lumen diameter $)$ and right common iliac artery diameter of $3.4 \mathrm{~cm}$ (2.6 cm lumen diameter). (a. = artery)

\subsection{Meshing}

We constructed the volume mesh within STAR-CCM+ (v9.04) (CD-adapco Group) using a core polyhedral mesh and a prism-layer mesh in the boundary layer that became progressively refined approaching the wall. The thickness of the prism-layer mesh and the surface size (edge length) were defined relative to the local lumen diameter so that the smaller arteries were well discretised. Any areas that were expected to have rapid changes in velocity (i.e. bifurcations) were also subject to refinement. The mesh can be seen in Figure 2.

In order to determine a sufficient level of (uniform) mesh refinement (number of prism-layers and polyhedra density coefficient), we applied the Grid Convergence Index (GCI) [50, 51] to steady-state simulations using the peak systolic flow conditions (i.e. peak flow in Figure 4). Figure 3 below shows the grid convergence of inlet pressure, mean WSS across the aneurysm and velocity probes scattered throughout the geometry. The GCI of all tested variables was below $2 \%$ and as this is considered to be a sufficient minimisation of the spatial discretisation error [5], no further mesh refinement was performed. The finest and chosen mesh (see Figure 2) contained 16 prism-layers and had a total mesh-cell count of 5.87 million.

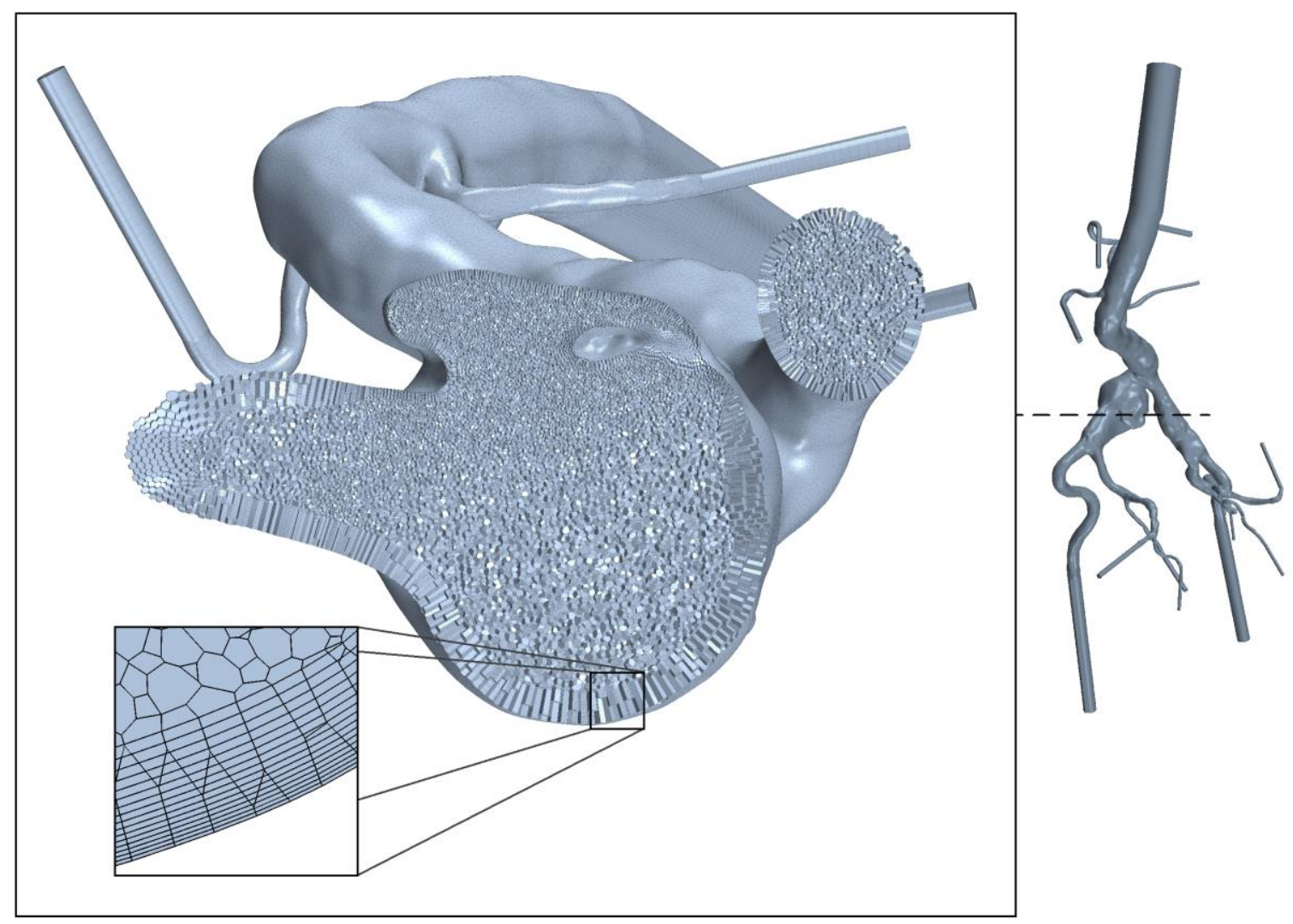

Figure 2: Mesh cross-section through the CIAA 'pocket', highlighting the prism-layer mesh and local mesh refinement. 


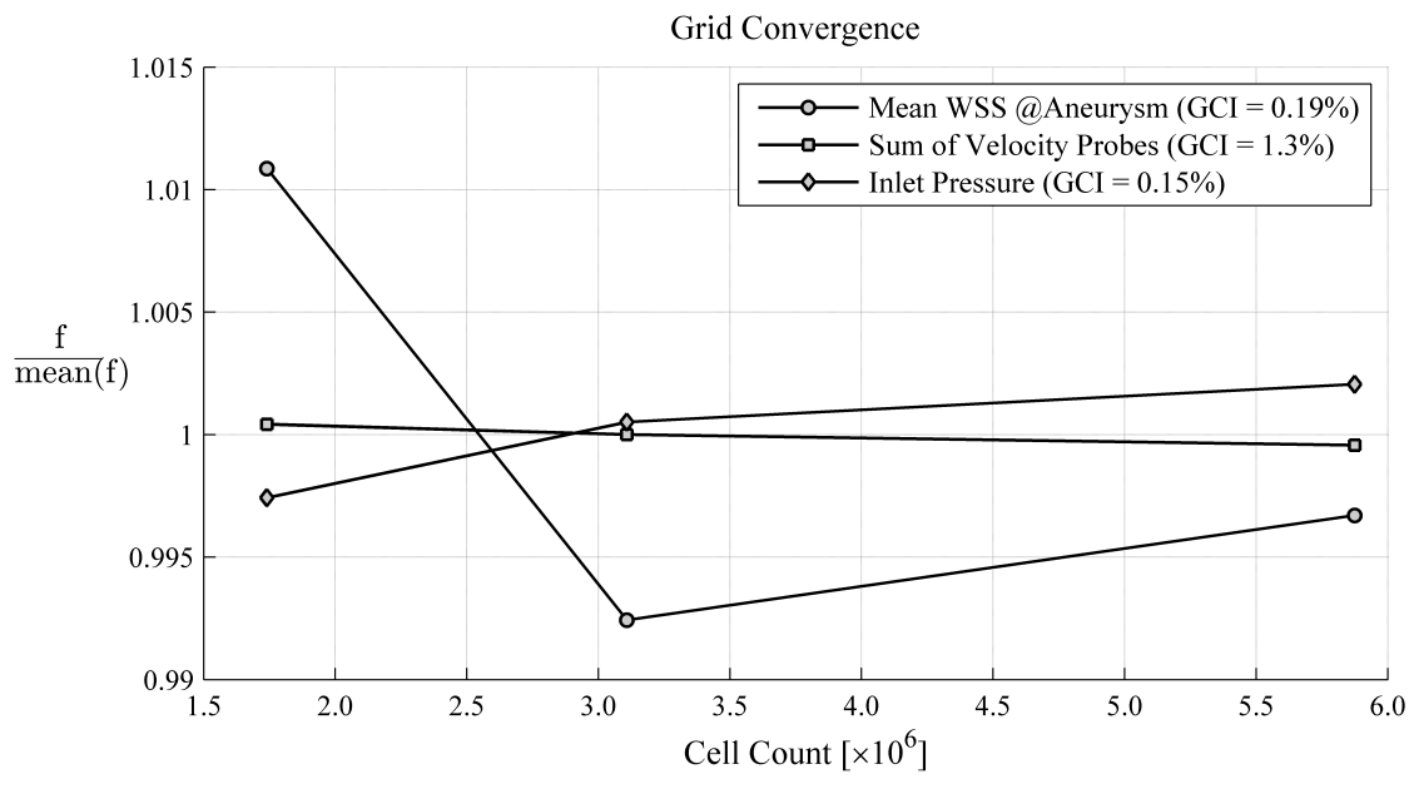

Figure 3: The normalised grid convergence of mean WSS across the CIA, velocity probes and inlet pressure plotted against the number of mesh-cells in the model domain. The results of the GCI calculations are included in the legend.

\subsection{Physical assumptions and boundary conditions}

The flow is expected to be turbulent within the patient's aorta and aneurysmal regions during latesystole; however it is also expected to largely relaminarize during diastole and early systole [35]. Reynolds-averaged turbulence models may be used to simulate turbulence efficiently on coarse meshes; though there is strong evidence that they are not suitable for pulsatile, relaminarizing flows in cardiovascular research $[52,53]$. With this in mind, we approximated the blood flow as laminar and considered the blood to be an incompressible fluid with a density of $1050 \mathrm{~kg} / \mathrm{m}^{3}$. The walls of the arteries were characterised by no-slip, rigid wall boundary conditions $[5,6,32,35,54,55]$ and the viscosity was modelled using a non-Newtonian approximation (Carreau-Yasuda, as implemented by Biasetti et al. [44]; [56]). By using a non-Newtonian model, as opposed Newtonian, we can capture the macro-scale shear-thinning of the blood, allowing a one-way Lagrangian particle transport model to provide a good prediction of individual blood-cell trajectories within the continuous phase (blood). This methodology follows that of Basciano et al. where the particleparticle and particle-fluid interactions are ignored [45] and is discussed further in section 2.5.

For the inlet boundary condition (SC aorta), we applied a mass flow waveform derived from volumetric flow data by Les et al. [57] (see Figure 4), where the waveform was scaled to suit the patient's age-estimated fat-free body mass using the allometric scaling relationships provided by Les et al. [57]. 
Figure 4: Top left: SC inlet waveforms and the patient's systolic and diastolic blood pressures. Top right: An example of the Windkessel Model used at each outlet boundary, where $\mathrm{Z}$ is the proximal resistance, $\mathrm{R}$ is the distal resistance and $\mathrm{C}$ is the vascular compliance. $\mathrm{P}_{\mathrm{atm}}$ is atmospheric pressure. Bottom: IR flow waveforms - the modelled waveform closely matches the data of Les et al. [57].

We explicitly coupled the 3D CFD simulation with a Windkessel model (RCR circuit) at each outlet boundary (see Figure 4) in order to approximate the resistance and compliance of the downstream vascular beds. This improves the estimation of pressure throughout the domain and allows the pressure waveform at the SC inlet to comply with the patient's measured systolic and diastolic pressures [57]. Our Windkessel parameters are calibrated according to previous methodology [35, 58]; with $30 \%$ of the common iliac flow passing through to the internal iliac artery. Furthermore, downstream of each internal iliac artery, the flow leaving the domain was split (directly) proportional to the area of each outlet boundary and the shape of the flow waveform leaving each of the iliac outlets was configured to resemble the infrarenal (IR) aortic waveform. This occurs as the compliance values used at each outlet are proportionate to the mean flow, and a proximal to distal resistance ratio of 5.6\% [58] is used at these outlets; similar to [35]. These assumptions are necessary, as patient-specific flow and pressure data for each outlet were not collected. Figure 4 shows the calculated IR flow waveform and how it compares with the IR flow data from the study by Les et al. (from which the inlet waveform was derived) [57]. 


\subsection{TAWSS, OSI and ECAP}

The site specificity of thrombus distribution is compared with the TAWSS and OSI fields, as well as the endothelial cell activation potential (ECAP). The ECAP is the ratio between the OSI and TAWSS, and is used to characterise the artery wall's degree of 'thrombotic susceptibility' in one index [36]. We know from previous work that artery wall regions of TAWSS below $0.36 \mathrm{~Pa}$ expect monocyte/cell adhesion to occur [47, 59-61] and the OSI describes maximum WSS vector oscillation, and atheroprone behaviour, at a value of 0.5 [34, 62]. Considering this, the threshold of ECAP describing critical thrombotic susceptibility is inferred as regions close to and above $1.4 \mathrm{~Pa}^{-1}$. The equations for TAWSS, OSI and ECAP are:

$$
\begin{gathered}
\text { TAWSS }=\frac{1}{T} \int_{t-T}^{t}|\boldsymbol{W S S}| d t \\
O S I=\frac{1}{2}\left(1-\frac{\left|\frac{1}{T} \int_{t-T}^{t} \boldsymbol{W S S} d t\right|}{\frac{1}{T} \int_{t-T}^{t}|\boldsymbol{W S S}| d t}\right) \\
E C A P=\frac{O S I}{T A W S S}
\end{gathered}
$$

where,

$\boldsymbol{W S S}$ is the instantaneous WSS vector,

$t$ is the instantaneous time, and

$T$ is the integration period (one or more cardiac cycles).

We calculated the TAWSS and OSI for 100 intervals per cardiac cycle and once the boundary waveforms converged (and any initial transience was not present) these results were computed for 10 cardiac cycles. Averaging for 10 cardiac cycles is traditionally quite conservative with many studies taking these fields over three or five cycles (i.e. [5, 35, 36]). However, in light of a recent study by Poelma et al. [54], we investigated the statistical convergence of these variables, as it had become clear that the systolic pulses were creating fluctuations within the CIAA that continued through diastole. When similar behaviour was present in a model of Poelma et al. [54], they showed that 28 cycles of data did not lead to complete convergence at a particular location. However, in our study the convergence of the results was not so slow and a 10 cardiac cycle averaging was sufficient. Regarding the convergence of the TAWSS field across the CIAA, the relative error between a 10cycle average and a nine-cycle average is $0.2 \%$.

\subsection{One-way Lagrangian particle modelling}

A one-way Lagrangian methodology was used to model blood-cell trajectories through the iliac arteries. We injected platelets and monocytes into the flow field on a transverse-plane within the IR aorta, well upstream of the common iliac arteries (see Figure 5). We assumed these particles to be spherical with diameters of 2 and $16 \mu \mathrm{m}$, and densities of $1040 \mathrm{~kg} / \mathrm{m}^{3}$ and $1070 \mathrm{~kg} / \mathrm{m}^{3}$ for the platelets [45] and monocytes [47], respectively. The particles were injected individually in parcels at randomly chosen spatial points (on the plane) with the same velocity as the continuous phase. The number of particles injected per time-step was proportional to the instantaneous mass flow through the IR aorta, and the forces acting on each particle were the pressure gradient force (Eq. 3d) and the drag force (Eq. 3b \& Eq. 3c). Following [45], $C_{d}$ (Eq. 1e) was the empirically-derived the Schiller-Naumann drag force coefficient, which varies for the transition between viscous and inertial particle transport.

$$
\begin{gathered}
F_{d}=\frac{1}{2} C_{d} \rho A_{p}\left|\boldsymbol{v}_{s}\right| \boldsymbol{v}_{\boldsymbol{s}} \\
C_{d}=\frac{R e_{p}}{24}\left(1+0.15 R e_{p}^{0.687}\right)
\end{gathered}
$$




$$
F_{p}=-V_{p}\left(\nabla P_{s}\right)
$$

where,

$R e_{p}$ is the particle Reynolds Number,

$\rho$ is fluid density,

$\boldsymbol{v}_{\boldsymbol{s}}$ is the particle slip velocity,

$A_{p}$ is the projected particle area,

$V_{p}$ is particle volume, and

$\nabla P_{S}$ is the gradient of static pressure.

Particle-wall collisions were considered linear-elastic, but with a wall adhesion probability $\left(A_{p}\right)$ equal to the near-wall-PRT limiter (monocyte adhesion efficiency function) introduced by Hardman et al. [47] (Eq. 2a):

$$
A_{p}=\frac{(T A W S S-0.4)^{2}}{2.4 T A W S S+0.16}
$$

where, the TAWSS must be less than $0.36 \mathrm{~Pa}$ for any wall adhesion to occur.

The particles were injected for ten cardiac cycles, starting from a well-developed flow-field (15 precomputed cycles), with $2 \times 10^{4}$ platelets and $2 \times 10^{4}$ monocytes injected per-cycle. While the concentrations of both platelets and monocytes are not physically realistic, they are sufficient to test hypotheses of ILT formation [45]. The residence time of the particles are analysed here using the residence time of the particles in the entire domain, rather than local sub-domains. Therefore a particle's residence time is considered equivalent to the particle's age (and does not follow the more common assessments of residence time [63]). A cell-based residence time was considered which measured the minimum time spent by particles in the computational cells [63]. However, it was more computationally efficient (and effective) to view residence time as an average of particle age in each cell (possible due to the minimal-bias introduction of the particles upstream of each iliac artery). Henceforth, in order to provide a comprehensive evaluation of how residence time varies throughout the geometry, for each of the modelled platelets and monocytes, we averaged the particle age at the nearest mesh-cell centre for 40 evenly spaced time intervals spanning the final two cycles. This is referred to as mean domain residence time (DRT).

\subsection{Numerical solvers, simulation details and particle trajectory validation}

The Navier-Stokes equations were solved using STAR-CCM+'s finite-volume discretisation and implicit-unsteady, segregated flow solver. STAR-CCM+ implements a 'SIMPLE algorithm' when the flow is segregated to control the solution update and enforce mass conservation with each timestep [64]. We used the second-order up-wind convection scheme and the temporal discretisation was second order [65], with $10^{3}$ time-steps spanning each cardiac cycle and a minimum of 15 inner iterations per time-step. A convergence condition was implemented, requiring the convergence of the normalised [65] continuity and momentum residuals to remain below $10^{-3}\left(\sim 10^{-9}\right.$ absolute). However, additional inner iterations were seldom needed (only during the peak of the systolic phase).

We performed our simulations using all $12 \mathrm{CPU}$ cores of a workstation with two Intel Xeon X5650 processors and each cardiac cycle took approximately 24 to 48 hours, depending on the number of particles injected. The track file for the particle trajectories and monitored values reached $9 \mathrm{~GB}$. 
STAR-CCM+'s Lagrangian implicit-unsteady solver was used to evaluate the particle trajectories for each time-step. As the one-way solution to the particle trajectories does not affect the Eulerian solution of the continuous phase, we only evaluated the particle trajectories at the last (Eulerian) iteration of each time-step. This unsteady approach follows the discrete element and discrete particle methods discussed in [66]. The local time-steps used to march the particles are calculated dynamically so that the temporal error becomes negligible [65], where these local time-steps are also bounded by minimum and maximum values. The first upper bound is the Eulerian time-step and following this the maximum local time-steps are also bound by the momentum relaxation time-scale used in the drag force model. Additionally, we imposed maximum and minimum Courant number $(C)$ bounds, limiting the transport of particles (parcels) according to the characteristic length of each particle (parcel) $(\Delta x)$ and the maximum of the parcel $\left(v_{p}\right)$ or fluid $(v)$ velocity (Eq. $\left.2 b\right)$.

$$
\frac{C_{\min } \Delta x}{\max \left(\left|v_{p}\right|,|v|\right)} \leq \delta t_{p} \leq \frac{C_{\max } \Delta x}{\max \left(\left|v_{p}\right|,|v|\right)}
$$

where,

$$
\begin{aligned}
& C_{\text {min }}=0.05, \text { and } \\
& C_{\text {max }}=0.35 .
\end{aligned}
$$

To test the Lagrangian models, the particle trajectories and wall-deposition efficiency were investigated for a simple case: particle injection into steady flow through a $90^{\circ}$ circular-pipe bend to model the physical experiments done by Pui et al. [67]. Note, that because the flow was steady, after injection, the particles were marched until they were either deposited on the pipe wall or they left the domain. The geometry and material/fluid properties used here follow that of Basciano et al. for this case [68]. The diameter of these injected particles is varied and the wall-deposition efficiency is compared for different Stokes Numbers (the ratio of the particle characteristic stopping distance to the vessel's characteristic length). We observed very good agreement with both the experimental work of Pui et al. and the computational work of Basciano et al. For Stokes Numbers spanning 0.04 to 0.6 the mean relative error between the modelled deposition efficiencies and the experimental values were $15 \%$ for our implementation and $93 \%$ for Basciano et al. However, when ignoring Stokes Numbers below 0.1 , both numerical models performed similarly ( $29 \%$ error).

\section{RESULTS AND DISCUSSION}

\subsection{Particle residence and transport}

As the injection location lay upstream of the common iliac arteries, it took four cycles for particles/cells to progress downstream of these arteries - half a cycle more on the side with the large aneurysm. The minimum time spent per-particle within each common iliac artery was approximately one and a half cycles, and after seven cycles each common iliac artery appeared densely populated with both monocytes and platelets. As shown in Figure 5, the net influx of particles continued to grow for both common iliac arteries and by the tenth cycle, the left side artery with the large aneurysm harboured approximately $6 \times 10^{4}$ monocytes and $6 \times 10^{4}$ platelets. In contrast, the other common iliac artery had half as many particles enter; however, it managed to entrap a similar portion of these particles. Regarding this, the ratio of maximum diameter to inlet diameter for both common iliac arteries is similar, at approximately 1.9. However, the inlet to the left CIAA is much greater than the inlet to the other common iliac artery, and has a vortex spanning its entrance (discussed later; see Figure 8). This vortex is possibly assisting the greater particle transport into the large aneurysm. 


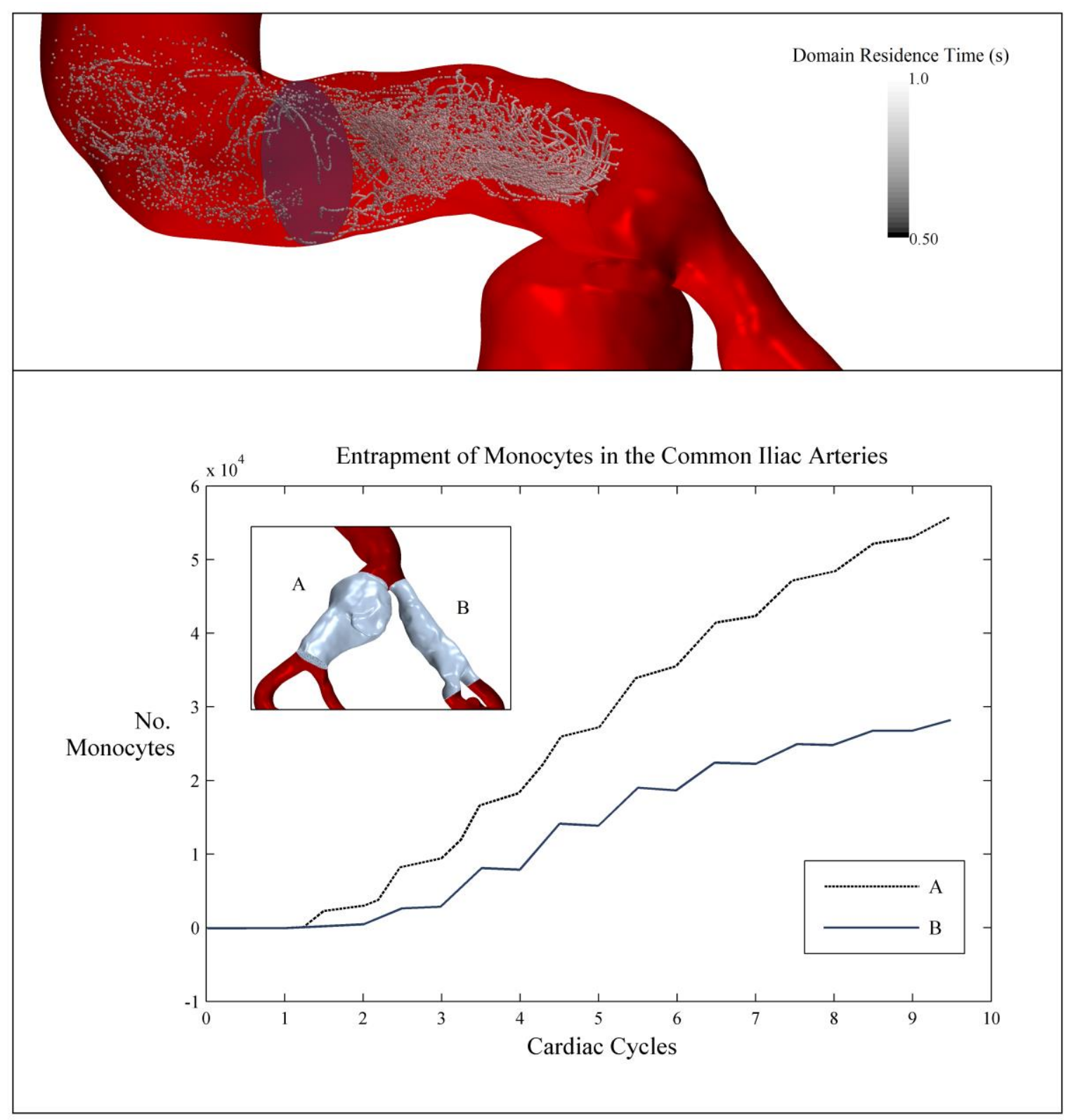

Figure 5: Top: Particles being injected for the first cycle in the IR aorta. Bottom: Particle count in the common iliac arteries since injection.

We observed little difference between the monocyte and platelet trajectories. Because of their small physical sizes, both cell-types have low momentum response times and respond promptly to changes in the flow field. The suspension of monocytes and platelets occurred in both common iliac arteries (upon the particle slip velocity reaching zero). When this happens there is no difference in the immediate residence of either cell-type at a particular location, as they both follow the carrier-fluid. In addition, a negligible amount of cell adhesion occurred throughout the model.

In the left CIAA, the mean DRT of both monocytes and platelets was elevated near the wall in the regions of significant ILT formation (almost everywhere). As shown in Figure 6, the lower and/or anterior portion of the aneurysm sac where the most ILT forms is associated with a large region of high mean DRT and cell entrapment. Another primary observation is the complete entrapment of cells within the pocket on the anterior side of the aneurysm (highest mean DRT). 


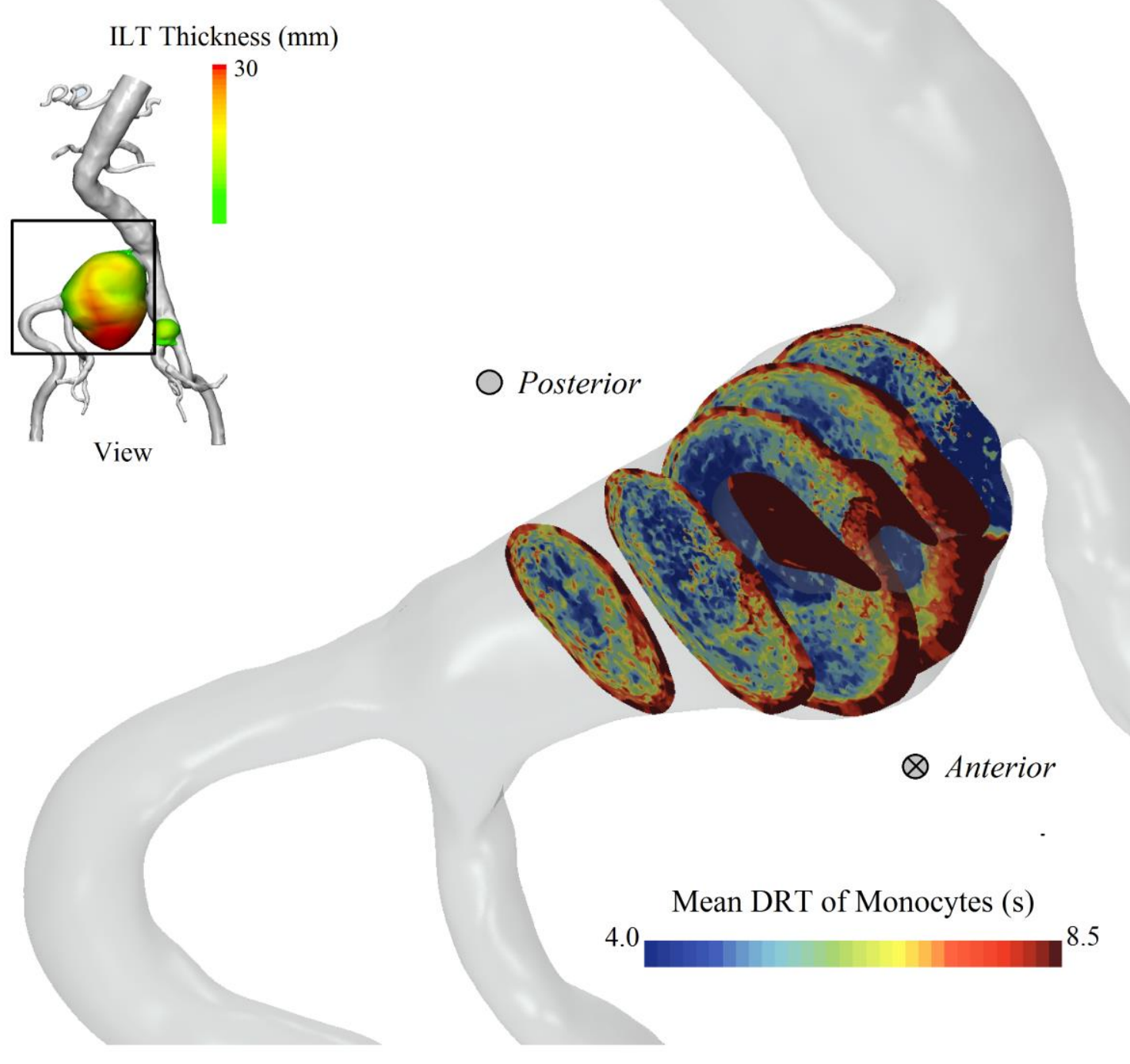

Figure 6: Plane sections showing the mean DRT of monocytes within the large left CIAA and colourcoded ILT thickness (top left).

There was reasonable agreement between the mean DRT and ILT location in the right contralateral common iliac artery (see Figure 7). We observed the posterior side near the distal bifurcation to have the greatest amount of ILT present and high mean DRT. However, we also found the exterior of the posterior side to have high mean DRT and cell entrapment, despite having no localised ILT formation. The mean DRT on the anterior side is more diffuse, with less ILT observed on CT. Our findings also show that the mean DRT is higher in regions where the artery is locally expanding - with some high mean DRT in the upstream, despite an absence of ILT. Future ILT formation may be expected in these regions; however, the absence of follow up data means that, in this instance, these regions show disagreement with expectations of ILT specificity. 

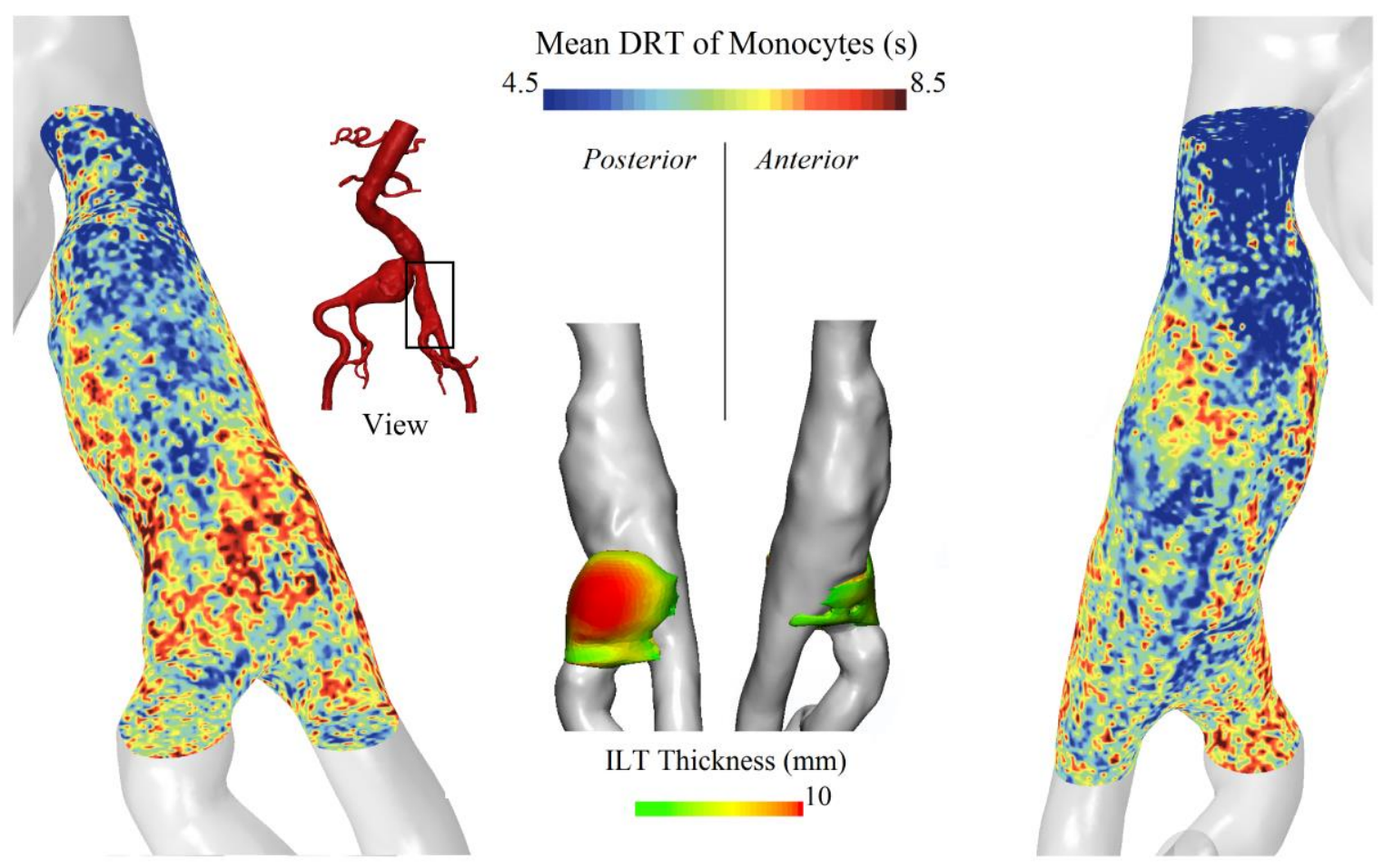

Figure 7: Posterior and anterior views of ILT and mean DRT in the less-diseased common iliac artery. This is a volume rendering of mean DRT showing the field value in the surface-adjacent mesh-cell which well represents the internal distribution of mean DRT in the fluid boundary layer.

\subsection{Velocity fields}

We found clear differences in the velocity fields between the two arteries, which can be attributed to the geometric differences. In the left CIAA, the inlet/neck angle to the upstream aorta is only slightly greater than that of the other common iliac, yet the inlet diameter is much greater $(\sim 2 x)$. We observed a vortex structure moving across the entrance to the left CIAA, which formed during systole at a point of flow separation on the top lip (see Figure 8a). This vortex structure leaves the aneurysm entrance and travels downward toward the anterior region of the aneurysm sac where it disperses when the net direction of flow reverses. Similar to the postulation by Biasetti et al. [40], this structure, which meets the $\lambda_{2}$ criterion of a vortex [69], carries with it many of the platelets and monocytes into the lower/anterior regions of the aneurysm sac where the majority of ILT has formed (see Figure 8). This behaviour occurs due to the low stokes number of the particles: they lack the inertia necessary to leave, or travel unaffected by coherent vortices. In this CIAA, we also found a permanent stagnation of flow within the pocket on the interior side and extremely low flow in the anterior region of the aneurysm sac. These regions, which are the most diseased/abnormal, are not flushed out during systolic flow. Furthermore, whilst the particles migrate to these regions of low flow, it takes longer for them to get close to the walls as they encounter significant deceleration. It required ten cycles for the concentration of platelets and monocytes within $1 \mathrm{~cm}$ of the aneurysm's lower anterior surface ( $9.5 \%$ of aneurysm volume) to reach the average concentration within the aneurysm. The flow stagnation and associated low TAWSS in this region lead to a high wall-cell adhesion probability. However, the inability for particles to reach the wall results in a negligible amount of wall-cell adhesion occurring in this model. 

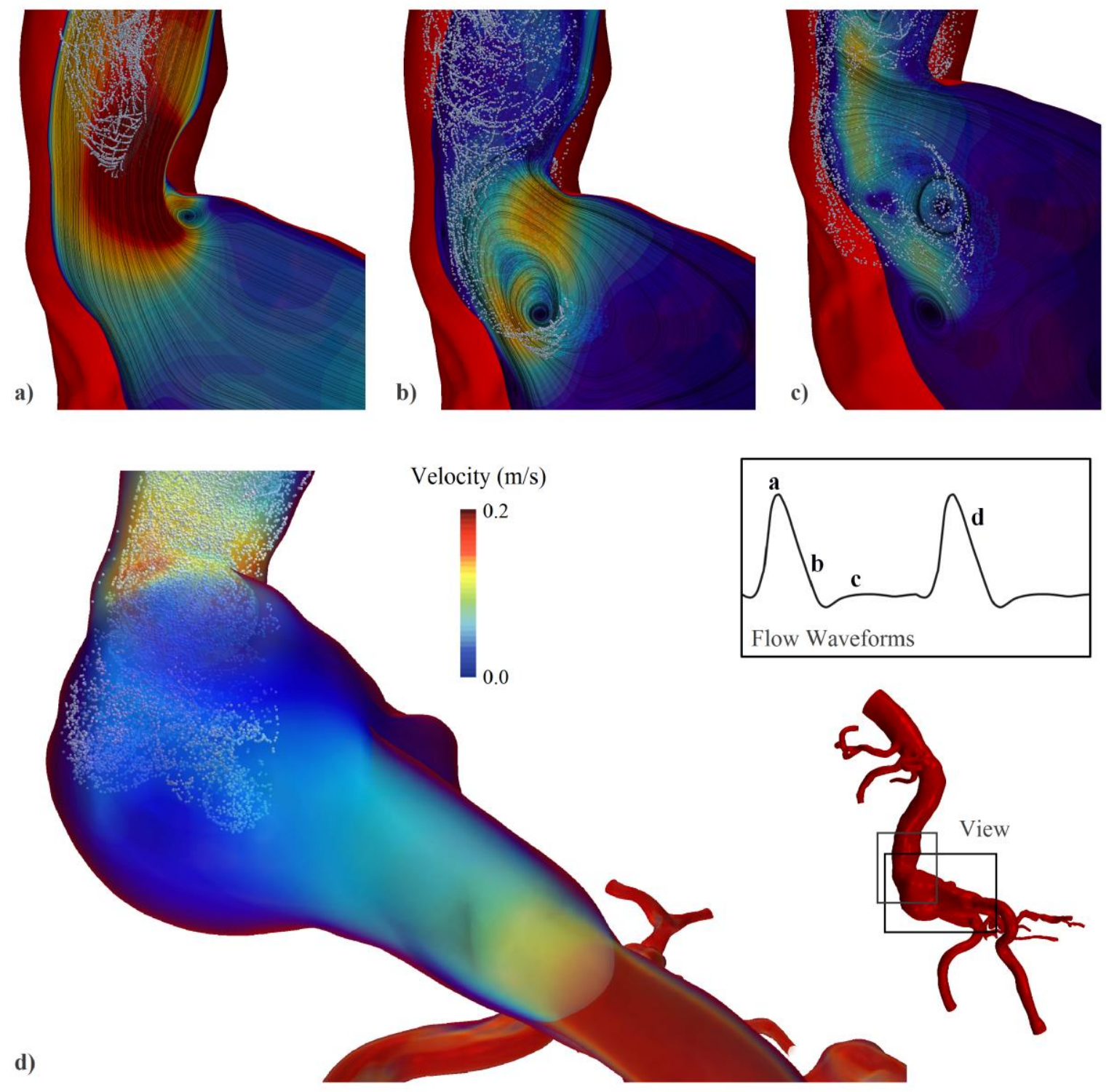

Figure 8: Platelets entering the left CIAA for four time-points ( $a, b, c$ and d) within the second and third cardiac cycles (since particle injection). Images (a), (b) and (c) show plane-constrained velocity streamlines through the aneurysm neck, while image (d) shows a volume rendering of the velocity field. This figure highlights the production of a vortex at the inlet to the aneurysm during systole (images $a$ and $b$ ) and how this structure progresses toward the lower portion of the aneurysm during diastole, ultimately diverting entering particles toward the aneurysms lower, heavily ILT-burdened region. This behaviour is similar to the previous hypothesis on the formation of ILT in aortic aneurysms (due to the observed vortex transport) [40].

Figure 9 shows the key stages of the velocity field within the right common iliac artery. Over the course of the cardiac cycle significant rotary motion in the freestream only occurred during late diastole, after flow reversal. The flow completely re-aligned itself during the systolic phase and sees an efficient transport of blood distally during this period. During systole, vortices formed where the artery begins to dilate at the proximal end. While these vortices did not exist for all phases of the cardiac cycle, as they progressed downstream they appeared to encourage particle transport toward the wall. There were a number of low velocity recirculation zones that were well developed at the end of systole near the regions where the ILT has formed in the distal of the artery (see Figure 9c and 9d). 


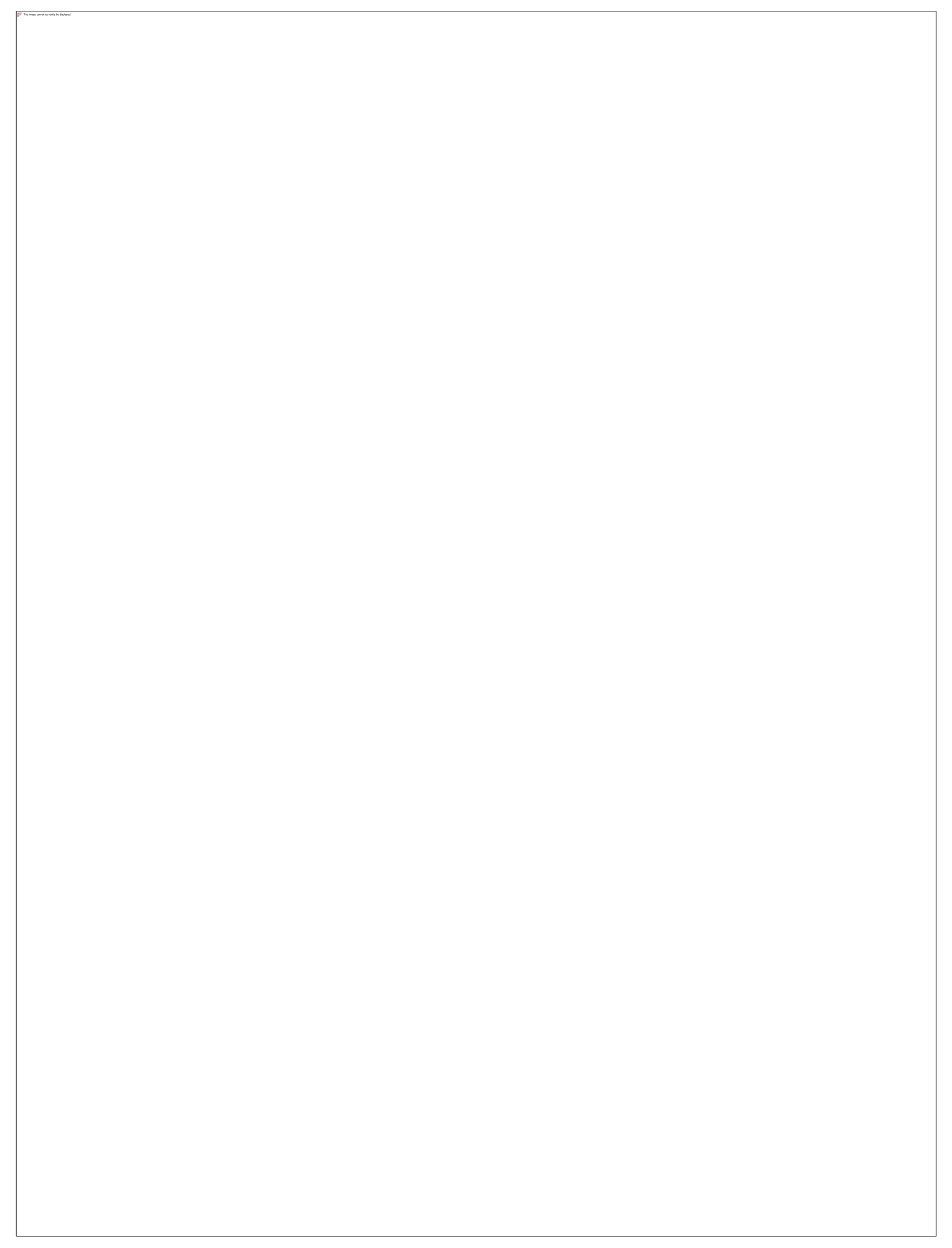

Figure 9: The plane tangential velocity field with constrained streamlines in centre of the lessdiseased common iliac artery for four time points (a, b, c and d). Image (a) shows the velocity field during the systolic phase. Image (b) shows the velocity field during systolic deceleration and the development of a vortex where the artery begins to dilate. Image (c) shows the end of the systolic phase and the development of low-velocity vortices near the distal bifurcation. Image (d) shows the velocity field as the net transport of blood through the artery reaches zero. 


\subsection{Wall shear stress fields and thrombus susceptibility}

We found extremely low TAWSS (expected cell adhesion) and a high OSI across the large aneurysm; in particular, within the 'pocket' (see Figure 10). There were large differences in both the TAWSS and OSI fields on the upper/posterior and lower/anterior surfaces of the aneurysm, correlating with regions of ILT. This is represented clearly by the ECAP, as the majority of mesh-cell faces on the lower/anterior surface are above the estimated value for critical thrombus susceptibility $\left(1.4 \mathrm{~Pa}^{-1}\right)$. Furthermore, the aneurysm's pocket, for all indices, was found to be the most thrombus susceptible region, as it sees the largest ECAP as well as the largest mean DRT, and flow stagnation. However, bulk transport of platelets and monocytes to that region did not occur, as most of the modelled particles more readily resided in other regions of the aneurysm where the flow was less stagnant, though still providing the necessary conditions for entrapment, i.e. in the lower/anterior of the sac. This may explain the lack of more significant thrombus close to the pocket (ILT thickness is $\sim 10 \mathrm{~mm}$ near the pocket).

Figure 10: TAWSS, OSI and ECAP fields shown for both the posterior/top and anterior/bottom of the common iliac artery with the large aneurysm.

In the other common iliac artery, the ECAP field showed some agreement with the site specificity of ILT (see Figure 11). The region near the bifurcation experienced low enough WSS for expected cell adhesion and we found a large patch of high OSI on the posterior surface of the artery where the majority of ILT formed. However, in the proximal region of this artery, where no ILT developed, there were some similar trends in the WSS fields (and some aneurysmal development). Though the absence of low-velocity, high-residence time recirculation zones in the upstream may explain why ILT formed in the distal portion of the artery, which would agree with the previous hypothesis of ILT formation in the distal sac [40].

Our results would be more conclusive, and less subjective, if baseline and/or follow up data were available for analysis. Although it remains clear that the CIAAs in this patient present similar values for the haemodynamic metrics at the locations of thrombotic built up, being proportional to the amount of thrombus developed in these regions. 
Figure 11: TAWSS, OSI and ECAP fields shown for both the posterior and anterior of the lessdiseased common iliac artery.

\section{LIMITATIONS}

Firstly, we have only investigated one case and we need to extend our study to include more patients with different geometric variations. Notably, the lack of any healthier, baseline data limits the interpretation of the results. Secondly, regarding the physics of our simulation, without a near-wall lift force [46, 47], the transport of particles away from (and toward) the wall may be lacking, and some of the particles could become artificially stuck in the near-wall boundary layer. However, as it took a long time for particles to penetrate into lower velocity regions, when considering ILTassociated flow stagnation (and particle suspension), wall-particle interaction may be reasonably expected, and therefore a superior near-wall model may simply confirm these expectations. The physical models used here lack the chemical cues and blood cell to wall recruitment mechanisms that are present, in vivo.

The time frame over which the results are gathered strongly affects observed particle behaviour and the measured residence times. Ten cycles of data may not be sufficient to gain a proper understanding of particle transport in larger aneurysms. However, residence time information may be gained with more computational ease if the particles were seeded within the areas of interest, or by considering fluid residence through the transport of a passive scalar - in the absence of particle modelling. Nevertheless, the particle modelling approach we used here remains a promising way to understand, develop and test hypotheses about ILT formation in aneurysms. A more comprehensive representation of PRT may be implemented using existing cell-based methods [63] -requiring a greater concentration of particles in order to (accurately) measure the time spent by particles in each computational cell (i.e. mean exposure time $[63,70])$.

The ECAP and associated WSS fields are limited as they only provide a measurement of thrombotic susceptibility, and further information about the flow field and particle history remains critical to the analysis of ILT formation. The methodology used in this study to assess the site specificity of ILT may be simplified. The recent work of Di Achille et al. [36] further scales the ECAP by the platelet activation potential recently proposed by Shadden \& Hendabadi [71] to calculate a new thrombus formation potential. By combining information on the flow-induced shear history of wall-proximal platelets with information on the thrombotic susceptibility (ECAP) of the endothelial layer, a single, simple measure can be produced. 


\section{CONCLUSION}

We examined the residence of monocytes and platelets within a patient's two common iliac arteries. As there are two common iliac arteries, when they have different levels of aneurysmal dilation and ILT burden there is an excellent opportunity to compare and contrast the haemodynamic stimuli associated with these diseases. This type of comparison cannot be done when solely studying AAA. Because of this, further study of the haemodynamics in the common iliac arteries has the potential to improve our understanding of aneurysm growth and ILT development.

We have presented a comprehensive method of simulating particle dispersion, and have correlated residence of particles to the site of ILT. We also compared regions of ILT to the behaviour of the velocity field, TAWSS, OSI and ECAP. We have shown that high particle residence time, flow stagnation, vortex transport and high ECAP all correlate spatially with the regions where ILT developed. This trend was observed in the patient's left CIAA and can be seen as an emerging trend in the contralateral common iliac artery. Our findings show some support for the previous hypotheses of thrombus formation [40] and convey that, in this case at least, the haemodynamic indicators correlated with regions of thrombus build-up, as well as the sustained development and growth of the thrombus. However, these conclusions would be more substantive if baseline and/or follow up data were available to confirm them.

\section{ACKNOWLEDGMENT}

We would like to sincerely thank CD-adapco for their generous support of our research. We would like to also thank the National Health and Medical Research Council (Grants APP1063986 and APP1083572) and the Australia Postgraduate Award. 


\section{REFERENCES}

1. Svensjö, S., et al., Low Prevalence of Abdominal Aortic Aneurysm Among 65-YearOld Swedish Men Indicates a Change in the Epidemiology of the Disease. Circulation, 2011. 124(10): p. 1118-1123.

2. Norman, P.E., et al., The anatomical distribution of iliac aneurysms: Is there an embryological basis? European Journal of Vascular and Endovascular Surgery, 2003. 25(1): p. 82-84.

3. Norman, P.E. and J.T. Powell, Site specificity of aneurysmal disease. Circulation, 2010. 121(4): p. 560-8.

4. Huang, Y., et al., Common iliac artery aneurysm: expansion rate and results of open surgical and endovascular repair. Journal of Vascular Surgery, 2008. 47(6): p. 12031210.

5. Doyle, B.J., et al., From Detection to Rupture: A Serial Computational Fluid Dynamics Case Study of a Rapidly Expanding, Patient-Specific, Ruptured Abdominal Aortic Aneurysm, in Computational Biomechanics for Medicine. 2014, Springer New York. p. 53-68.

6. Steinman, D.A., et al., Variability of computational fluid dynamics solutions for pressure and flow in a giant aneurysm: the ASME 2012 Summer Bioengineering Conference CFD Challenge. Journal of Biomechanical Engineering, 2013. 135(2): p. 021016.

7. Tong, J. and G.A. Holzapfel, Structure, Mechanics, and Histology of Intraluminal Thrombi in Abdominal Aortic Aneurysms. Annals of Biomedical Engineering, 2015. 43(7): p. 1488-1501.

8. Sun, Z. and L. Xu, Computational fluid dynamics in coronary artery disease. Computerized Medical Imaging and Graphics, 2014. 38(8): p. 651-663.

9. Doyle, B.J., et al., Vessel asymmetry as an additional diagnostic tool in the assessment of abdominal aortic aneurysms. Journal of Vascular Surgery, 2009. 49(2): p. 443-54.

10. Doyle, B.J., et al., Regions of high wall stress can predict the future location of rupture of abdominal aortic aneurysm. Cardiovascular and Interventional Radiology, 2014. 37(3): p. 815-8.

11. Gasser, T.C., et al., Biomechanical rupture risk assessment of abdominal aortic aneurysms: model complexity versus predictability of finite element simulations. European Journal of Vascular and Endovascular Surgery, 2010. 40(2): p. 176-85.

12. Pijls, N.H.J., et al., Measurement of Fractional Flow Reserve to Assess the Functional Severity of Coronary-Artery Stenoses. New England Journal of Medicine, 1996. 334(26): p. 1703-1708.

13. Tonino, P.A.L., et al., Fractional Flow Reserve versus Angiography for Guiding Percutaneous Coronary Intervention. New England Journal of Medicine, 2009. 360(3): p. 213-224.

14. Blacher, J., et al., Aortic Pulse Wave Velocity as a Marker of Cardiovascular Risk in Hypertensive Patients. Hypertension, 1999. 33(5): p. 1111-1117.

15. Wilkinson, I.B., J.R. Cockcroft, and D.J. Webb, Pulse wave analysis and arterial stiffness. Journal of Cardiovascular Pharmacology, 1998. 32 Suppl 3: p. S33-7.

16. Arzani, A. and S.C. Shadden, Characterization of the transport topology in patientspecific abdominal aortic aneurysm models. Physics of Fluids, 2012. 24(8): p. 081901.

17. Sabooni, H., K. Hassani, and H.G. Bahraseman, Modeling of Iliac Artery Aneurysm using Fluid-Structure Interaction. Journal of Mechanics in Medicine and Biology, 2015. 15(01): p. 1550041.

18. Harter, L.P., et al., Ultrasonic evaluation of abdominal aortic thrombus. Journal of Ultrasound in Medicine 1982. 1(8): p. 315-8. 
19. Houard, X., et al., Topology of the fibrinolytic system within the mural thrombus of human abdominal aortic aneurysms. The Journal of Pathology, 2007. 212(1): p. 208.

20. Swedenborg, J. and P. Eriksson, The intraluminal thrombus as a source of proteolytic activity. Annals of the New York Academy of Sciences, 2006. 1085: p. 133-8.

21. Vande Geest, J.P., et al., Towards a noninvasive method for determination of patientspecific wall strength distribution in abdominal aortic aneurysms. Annals of Biomedical Engineering, 2006. 34(7): p. 1098-106.

22. Speelman, L., et al., The mechanical role of thrombus on the growth rate of an abdominal aortic aneurysm. Journal of Vascular Surgery, 2010. 51(1): p. 19-26.

23. Hans, S.S., et al., Size and location of thrombus in intact and ruptured abdominal aortic aneurysms. Journal of Vascular Surgery, 2005. 41(4): p. 584-8.

24. Truijers, M., et al., In-vivo imaging of changes in abdominal aortic aneurysm thrombus volume during the cardiac cycle. Journal of Endovascular Therapy, 2009. 16(3): p. 314-9.

25. O'Leary, S.A., et al., The biaxial mechanical behaviour of abdominal aortic aneurysm intraluminal thrombus: Classification of morphology and the determination of layer and region specific properties. Journal of Biomechanics, 2014. 47(6): p. 1430-1437.

26. Labruto, F., L. Blomqvist, and J. Swedenborg, Imaging the Intraluminal Thrombus of Abdominal Aortic Aneurysms: Techniques, Findings, and Clinical Implications. Journal of Vascular and Interventional Radiology, 2011. 22(8): p. 1069-1075.

27. Salsac, A.V., S. Sparks, and J. Lasheras, Hemodynamic Changes Occurring during the Progressive Enlargement of Abdominal Aortic Aneurysms. Annals of Vascular Surgery, 2004. 18(1): p. 14-21.

28. Salsac, A.V., et al., Evolution of the wall shear stresses during the progressive enlargement of symmetric abdominal aortic aneurysms. Journal of Fluid Mechanics, 2006. 560: p. 19-51.

29. Lasheras, J.C., The Biomechanics of Arterial Aneurysms. Annual Review of Fluid Mechanics, 2007. 39(1): p. 293-319.

30. Raz, S., et al., DPIV Prediction of Flow Induced Platelet Activation-Comparison to Numerical Predictions. Annals of Biomedical Engineering, 2007. 35(4): p. 493-504.

31. Rayz, V.L., et al., Flow residence time and regions of intraluminal thrombus deposition in intracranial aneurysms. Annals of Biomedical Engineering, 2010. 38(10): p. 3058-69.

32. Boyd, A.J., et al., Low wall shear stress predominates at sites of abdominal aortic aneurysm rupture. Journal of Vascular Surgery, 2015: p. 7.

33. Lehoux, S., Y. Castier, and A. Tedgui, Molecular mechanisms of the vascular responses to haemodynamic forces. Journal of Internal Medicine, 2006. 259(4): p. 381-392.

34. Ku, D.N., et al., Pulsatile flow and atherosclerosis in the human carotid bifurcation. Positive correlation between plaque location and low oscillating shear stress. Arteriosclerosis, Thrombosis, and Vascular Biology, 1985. 5(3): p. 293-302.

35. Les, A.S., et al., Quantification of hemodynamics in abdominal aortic aneurysms during rest and exercise using magnetic resonance imaging and computational fluid dynamics. Annals of Biomedical Engineering, 2010. 38(4): p. 1288-313.

36. Di Achille, P., et al., A haemodynamic predictor of intraluminal thrombus formation in abdominal aortic aneurysms. Proceedings of the Royal Society A: Mathematical, Physical and Engineering Sciences, 2014. 470(2172): p. 20140163.

37. O'Rourke, M.J., J.P. McCullough, and S. Kelly, An investigation of the relationship between hemodynamics and thrombus deposition within patient-specific models of abdominal aortic aneurysm. Proceedings of the Institution of Mechanical Engineers, Part H, 2012. 226(7): p. 548-64. 
38. Arzani, A., et al., A longitudinal comparison of hemodynamics and intraluminal thrombus deposition in abdominal aortic aneurysms. American Journal of Physiology - Heart and Circulatory Physiology, 2014. 307(12): p. H1786-H1795.

39. Yamazumi Md, K., et al., An Activated State of Blood Coagulation and Fibrinolysis in Patients with Abdominal Aortic Aneurysm 1. The American Journal of Surgery, 1998. 175(4): p. 297-301.

40. Biasetti, J., F. Hussain, and T.C. Gasser, Blood flow and coherent vortices in the normal and aneurysmatic aortas: a fluid dynamical approach to intra-luminal thrombus formation. Journal of The Royal Society Interface, 2011. 8(63): p. 144961.

41. Guimaraes, T.A., et al., Morphological aspects of mural thrombi deposition residual lumen route in infrarenal abdominal aorta aneurisms. Acta Cirurgica Brasileira, 2008. 23 Suppl 1: p. 151-6; discussion 156.

42. Muraki, N., Ultrasonic studies of the abdominal aorta with special reference to hemodynamic considerations on thrombus formation in the abdominal aortic aneurysm. Journal of Japanese College Angiology, 1983. 23: p. 401-413.

43. Vega de Ceniga, M., et al., Analysis of expansion patterns in 4-4.9 cm abdominal aortic aneurysms. Annals of Vascular Surgery, 2008. 22(1): p. 37-44.

44. Biasetti, J., et al., An integrated fluid-chemical model toward modeling the formation of intra-luminal thrombus in abdominal aortic aneurysms. Frontiers in Physiology, 2012. 3: p. 266.

45. Basciano, C., et al., A relation between near-wall particle-hemodynamics and onset of thrombus formation in abdominal aortic aneurysms. Annals of Biomedical Engineering, 2011. 39(7): p. 2010-26.

46. Longest, P.W., C. Kleinstreuer, and J.R. Buchanan, Efficient computation of microparticle dynamics including wall effects. Computers \& Fluids, 2004. 33(4): p. 577601.

47. Hardman, D., et al., On the prediction of monocyte deposition in abdominal aortic aneurysms using computational fluid dynamics. Proceedings of the Institution of Mechanical Engineers, Part H, 2013. 227(10): p. 1114-24.

48. Wood, N.B., Aspects of Fluid Dynamics Applied to the Larger Arteries. Journal of Theoretical Biology, 1999. 199(2): p. 137-161.

49. Fitzgerald, T. Human Gross Anatomy Atlas. 2015 [cited 2015 1/05]; Available from: http://cias.rit.edu/ tpf1471/Fitzgerald_783/anatomy/pelvisSkeleton.html.

50. Roache, P.J., Perspective: A Method for Uniform Reporting of Grid Refinement Studies. Journal of Fluids Engineering, 1994. 116(3): p. 405-413.

51. Procedure for Estimation and Reporting of Uncertainty Due to Discretization in CFD Applications. Journal of Fluids Engineering, 2008. 130(7): p. 078001-078001.

52. Taylor, C. and D. Steinman, Image-Based Modeling of Blood Flow and Vessel Wall Dynamics: Applications, Methods and Future Directions. Annals of Biomedical Engineering, 2010. 38(3): p. 1188-1203.

53. Stonebridge, P.A. and C.M. Brophy, Spiral laminar flow in arteries? The Lancet, 1991. 338(8779): p. 1360-1361.

54. Poelma, C., P.N. Watton, and Y. Ventikos, Transitional flow in aneurysms and the computation of haemodynamic parameters. Journal of The Royal Society Interface, 2015. 12(105).

55. Wolters, B.J.B.M., et al., A patient-specific computational model of fluid-structure interaction in abdominal aortic aneurysms. Medical Engineering \& Physics, 2005. 27(10): p. 871-883.

56. Leuprecht, A. and K. Perktold, Computer simulation of non-newtonian effects on blood flow in large arteries. Computer Methods in Bbiomechanics and Biomedical Engineering, 2001. 4(2): p. 149-63. 
57. Les, A.S., et al., Supraceliac and Infrarenal Aortic Flow in Patients with Abdominal Aortic Aneurysms: Mean Flows, Waveforms, and Allometric Scaling Relationships. Cardiovascular Engineering and Technology, 2010. 1(1).

58. Laskey, W.K., et al., Estimation of total systemic arterial compliance in humans. Journal of Applied Physiology (Bethesda, Md. : 1985), 1990. 69(1): p. 112-9.

59. Lawrence, M.B., et al., Rolling of lymphocytes and neutrophils on peripheral node addressin and subsequent arrest on ICAM-1 in shear flow. European Journal of Immunology, 1995. 25(4): p. 1025-31.

60. Lawrence, M.B., L.V. McIntire, and S.G. Eskin, Effect offlow on polymorphonuclear leukocytelendothelial cell adhesion. Blood, 1987. 70(5): p. 1284-90.

61. Worthen, G.S., et al., Effects of shear stress on adhesive interaction between neutrophils and cultured endothelial cells. Journal of Applied Physiology (Bethesda, Md. : 1985), 1987. 63(5): p. 2031-41.

62. Ku, D.N., et al., Flow patterns in the abdominal aorta under simulated postprandial and exercise conditions: An experimental study. Journal of Vascular Surgery, 1989. 9(2): p. 309-316.

63. Shadden, S.C. and A. Arzani, Lagrangian Postprocessing of Computational Hemodynamics. Annals of Biomedical Engineering, 2015. 43(1): p. 41-58.

64. Fergizer, J.H. and M. Peric, Computational Methods for Fluid Dynamics. 3 ed. 2002: Springer: Berlin-Heidelberg. 426.

65. CD-adapco, STAR-CCM+ User Guide. 2014.

66. Crowe, C.T., et al., Droplet-Particle Cloud Equations, in Multiphase Flows with Droplets and Particles, Second Edition. 2011, CRC Press. p. 235-258.

67. Pui, D.Y.H., F. Romay-Novas, and B.Y.H. Liu, Experimental Study of Particle Deposition in Bends of Circular Cross Section. Aerosol Science and Technology, 1987. 7(3): p. 301-315.

68. Basciano, C., Computational particle-hemodynamics analysis applied to an abdominal aortic aneurysm with thrombus and microsphere-targeting of liver tumors, in Department of Mechanical and Aerospace Engineering. 2010, North Carolina State University.

69. Jeong, J. and F. Hussain, On the identification of a vortex. Journal of Fluid Mechanics, 1995. 285: p. 69-94.

70. Lonyai, A., et al., New Insights into Pacemaker Lead-Induced Venous Occlusion: Simulation-Based Investigation of Alterations in Venous Biomechanics. Cardiovascular Engineering, 2010. 10(2): p. 84-90.

71. Shadden, S. and S. Hendabadi, Potential fluid mechanic pathways of platelet activation. Biomechanics and Modeling in Mechanobiology, 2013. 12(3): p. 467474. 